\title{
Aliasing errors due to quadratic nonlinearities on triangular spectral / $h p$ element discretisations
}

\author{
Robert M. Kirby • Spencer J. Sherwin
}

Received: 25 December 2005 / Accepted: 19 July 2006 /

Published online: 3 October 2006

(C) Springer Science+Business Media B.V. 2006

\begin{abstract}
In this paper, consideration is given to how aliasing errors, introduced when evaluating nonlinear products, inexactly affect the solution of Galerkin spectral $/ h p$ element polynomial discretisations on triangles. A theoretical discussion is presented of how aliasing errors are introduced by a collocation projection onto a set of quadrature points insufficient for exact integration, and consider interpolation projections to geometrically symmetric collocation points. The discussion is corroborated by numerical examples that elucidate the key features. The study is first motivated with a review of aliasing errors introduced in one-dimensional spectral-element methods (these results extend naturally to tensor-product quadrilaterals and hexahedra.) Within triangular domains two commonly used expansions are a hierarchical, or modal, expansion based on a rotationally non-symmetric collapsed-coordinate system, and a Lagrange expansion based on a set of rotationally symmetric nodal points. Whilst both expansions span the same polynomial space, the construction of the two bases numerically motivates a different set of collocation points for use in the collocation projection of a nonlinear product. The purpose of this paper is to compare these two collocation projections. The analysis and results show that aliasing errors produced using a collocation projection on the rotationally non-symmetric, collapsed-coordinate system are significantly smaller than those for a collocation projection using the rotationally symmetric nodal points. In the case of the collapsed coordinate projection, if the Gaussian quadrature order employed is less than half the polynomial order of the integrand, then it is possible for the aliasing error to modify the constant mode of the expansion and therefore affect the conservation property of the approximation. However, the use of a collocation projection onto a polynomial expansion associated with a set of rotationally symmetric nodal points within the triangle is always observed to be non-conservative. Nevertheless, the rotationally symmetric collocation will maintain the overall symmetry of the triangular region, which is not typically the case when a collapsed coordinate quadrature projection is used.
\end{abstract}

R. M. Kirby

School of Computing, University of Utah, Salt Lake City, Utah, 84112, USA

e-mail: kirby@cs.utah.edu

S. J. Sherwin $(\varangle)$

Department of Aeronautics, Imperial College London,

South Kensington Campus, London, SW7 2AZ, UK

e-mail: s.sherwin@imperial.ac.uk 
Keywords Electrostatic points $\cdot$ Fekete points $\cdot$ High-order finite elements $\cdot$ Integration errors · Triangular Lobatto points

\section{Introduction}

In spectral methods, the quadratic nonlinearities of the incompressible Navier-Stokes equations and the cubic nonlinearities in the compressible Navier-Stokes are normally computed in physical space using collocation projections. Specifically, the primary fields (i.e., velocity, pressure, energy) are first transformed into a physical-space representation where the fields are discretely evaluated at a set of collocation points. In the frame of triangular spectral $/ h p$ methods, two reasonably commonly adopted types of polynomial expansions motivate different choices of collocation points. The first is a hierarchical or modal expansion discussed in Sect. 3.2, which is designed as a generalised tensor product using a collapsed-coordinate system which does not have rotational symmetry. The second expansion discussed in Sect. 3.3, is a nodal expansion utilising a Lagrange polynomial through a set of nodal points that are normally chosen to be rotationally symmetric in the triangle and have good interpolation properties. From an implementation point of view, the first expansion motivates the choice of collocation points based upon the Gaussian quadrature points evaluated along the non-symmetric collapsed coordinate system. Equivalently, the second basis motivates the use of the nodal points as collocation points due to the Kronecker-delta property of this expansion basis. Having determined potential collocation points, nonlinear products can be obtained at the discrete points in a collocation fashion analogous to the pseudo-spectral evaluation commonly adopted in global spectral methods.

A normal practice in polynomial Galerkin methods is the use of sufficient quadrature to integrate the linear differential terms exactly. In $[1,2]$ it was argued that employing an insufficient quadrature rule for evaluating nonlinear terms leads to an aliasing pollution that degrades the accuracy of the solution and in the worst case leads to numerical instability. In [2], the proposed solution was dubbed "over-integration" a term implying that de-aliasing of the solution requires the use of more quadrature points than would be necessary to integrate linear differential terms in a polynomial Galerkin method exactly. In this paper we refer to this technique as "insufficient quadrature" since this terminology more accurately represents the approximation. The errors caused by insufficient quadrature can be bounded by the theoretical estimates of [3], and often this result is used to support the idea that, if the simulation is well-resolved, then the numerical crimes committed by insufficient quadrature are negligible. However, as pointed out in [4], the theory does not address what happens in the marginally or badly resolved cases frequently encountered in practice.

Although use of consistent integration of the nonlinear products eliminates the aliasing problem due to insufficient quadrature for handling nonlinear terms, there is a computational penalty. Specifically, at least $3 / 2$ times more quadrature points per direction are required to properly integrate quadratic nonlinearities (two times more points per direction for cubic nonlinearities). Hence, there may be a reasonably high computational cost when adopting consistent integration rules for the nonlinear terms as compared to a quadrature rule designed to consistently handle the standard linear terms.

The numerical practitioner often wants to know: (1) When is exact or consistent integration absolutely necessary? (2) How does the aliasing error influence my solution? (3) Is there any computationally efficient middle-ground between insufficient quadrature and exact integration (for example, will evaluating the nonlinear products at a special set of points help minimize the aliasing)?

The main purpose of this paper is to understand aliasing errors generated when evaluating quadratic nonlinear products on spectral $/ h p$ triangle discretisations with the goal of helping the numerical practitioner answer the questions mentioned above. Specifically, we attempt to answer the following questions: 
- If one continues to evaluate nonlinear terms at the tensor-product quadrature points in collapsed coordinates, as is typical practice in modal spectral $/ h p$ element discretisations $[5,6]$, what is the effect on the modal energy?

- When insufficient quadrature is applied, does the geometrically non-symmetric nature of the tensorproduct quadrature points in collapsed coordinates end up being more harmful than helpful (i.e., does one trade accuracy for numerical efficiency)?

- If one were to evaluate nonlinear products at a geometrically symmetric collection of points (such as the Fekete [7], electrostatic points [8] or triangular Lobatto points [9] used in triangle collocation methods), and were then to evaluate the Galerkin approximation, would the aliasing error be less (or better distributed)?

The paper is organised as follows. In Sect. 2, we present a theoretical discussion of the aliasing error due to the projection of squared polynomials (to mimic quadratic nonlinearities) and provide a numerical example to help gain intuition into the results in higher dimensions. In Sect. 3, we present a review of tensor-product integration on triangles, and then present a theoretical discussion of aliasing when using a modal orthogonal basis or using a nodal collocation basis. In Sect. 4 we present numerical results to demonstrate and corroborate the theoretical results presented in Sect. 3. In Sect. 5, we summarize our findings and provide some guidelines for the numerical methods practitioner to understand the trade-offs between the different choices presented.

\section{Motivation}

To motivate our discussion, we examine the essence of the nonlinear evaluation based on a simple illustrative example in one dimension. Assume we are given a single spectral $/ h p$ element $E$ defined on $[-1,1]$ supporting polynomials up to degree $P$. We denote by $\left\{\phi_{i}(\xi)\right\}, i=0, \ldots, P$ an orthonormal basis in $L_{2}[-1,1]$ (i.e., the scaled Legendre-polynomial basis) that spans the polynomial space $\mathcal{P}^{P}$; the index $i$ provides the maximum degree of the polynomial expression denoted by the basis function $\phi_{i}$.

We now presume that we have a polynomial expansion of the form:

$u(\xi)=\sum_{i=0}^{P} \hat{u}_{i} \phi_{i}(\xi)$,

and we are interested in obtaining the expansion

$w(\xi)=\sum_{k=0}^{P} \hat{w}_{k} \phi_{k}(\xi)$

such that $\left\|w(\xi)-[u(\xi)]^{2}\right\|_{L_{2}}$ is minimized. The modal coefficients $\hat{w}_{k}$ are uniquely determined through the Galerkin projection which can be determined in this case by the following expression:

$\hat{w}_{k}=\sum_{i, j=0}^{P} \hat{u}_{i} \hat{u}_{j} \int_{-1}^{1} \phi_{i}(\xi) \phi_{j}(\xi) \phi_{k}(\xi) \mathrm{d} \xi$

for $k=0, \ldots, P$.

Note that the integrand in Eq. 1 contains the product of three polynomials from $\mathcal{P}^{P}$, and hence it is at most a polynomial in $\mathcal{P}^{3 P}$. For notational simplicity, let us define the inner product of two functions on $[-1,1]$ as follows:

$(f, g)=\int_{-1}^{1} f(\xi) g(\xi) \mathrm{d} \xi$, 
and the corresponding Gauss-Lobatto-Legendre (GLL) quadrature approximation of the inner product

$[f, g]_{Q}=\sum_{i=1}^{Q} \omega_{i} f\left(z_{i}\right) g\left(z_{i}\right)$,

where $z_{i}$ and $\omega_{i}$ denote the GLL quadrature points and weights, respectively. The natural number $Q$ denotes the number of points (or weights) used. We know that the GLL quadrature is an exact approximation (to machine precision) of the inner product when the integrand $\mathcal{I} \in \mathcal{P}^{2 Q-3}$ and has an error term of the following form [10,11],

$E_{Q}=-\frac{Q(Q-1)^{3} 2^{2 Q-1}[(Q-2) !]^{4}}{(2 Q-1)[(2 Q-2) !]^{3}} \mathcal{I}^{(2 Q-2)}(\zeta)$

where $\zeta \in[-1,1]$.

Now let us consider the case where an insufficient quadrature is employed. As a common example, we consider a quadrature order sufficient to integrate polynomials in $\mathcal{P}^{2 P}$, as typically required for a linear operation in a Galerkin discretisation. The relation between the true modal solution $\hat{w}_{k}$, defined in Eq. 1, and the approximate modal solution $\tilde{w}_{k}$, defined as

$\tilde{w}_{k}=\sum_{i, j=0}^{P} \hat{u}_{i} \hat{u}_{j}\left[\phi_{i}(\xi) \phi_{j}(\xi), \phi_{k}(\xi)\right]_{Q}$,

is given by the following expression,

$\tilde{w}_{k}=\hat{w}_{k}-\left[\sum_{\substack{i, j=0 \\ i+j>2 P-k}}^{P} \hat{u}_{i} \hat{u}_{j}\left(\left(\phi_{i} \phi_{j}, \phi_{k}\right)-\left[\phi_{i} \phi_{j}, \phi_{k}\right]_{Q}\right)\right]$,

for $k=0, \ldots, P$.

Remark 1 In the above summation, $\left(\phi_{i} \phi_{j}, \phi_{k}\right)=\left[\phi_{i} \phi_{j}, \phi_{k}\right]_{Q}$ for $i+j+k \leq 2 P$, leading to the lower bound. If the quadrature order is sufficient to integrate polynomials in $\mathcal{P}^{2 P}$ exactly (for instance, with $Q=P+2$ ), then the $k=0$ term (which represents the mean mode) is computed exactly. For each subsequent mode $(k=1, \ldots, P)$, the number of aliasing terms (in the right-most summation term) increases. For instance, $\tilde{w}_{1}$ obtains aliasing energy from the $(i=j=P)$ term only; $\tilde{w}_{2}$ obtains aliasing energy from the $(i=j=P)$ term, the $(i=P, j=P-1)$ term and the $(i=P-1, j=P)$ term.

The above remark can be used to explain two commonly used heuristics in nonlinear term evaluation. First, in a well-resolved simulation, the aliasing errors are inconsequential. If we take a well-resolved simulation to be one in which the modes of $u$ decay with a $\hat{u}_{k} \propto(1 / k)^{r}$ type fall-off for $(r>1)$, the aliasing effect is significantly reduced due to the dependence of the amount of aliasing energy on the modal coefficients of the function being squared (i.e., due to the decay of the $\hat{u}_{i} \hat{u}_{j}$ terms in Eq. 2 . The second heuristic is that a slight over-integration (i.e., adding a few extra quadrature points) can have a stabilizing effect, as seen in [12]. From Eq. 2 we see that slight over-integration can reduce or eliminate the aliasing error from the low modes-those modes on which standard linear diffusion acts most slowly. The net effect is a reduction of the aliasing error and most likely better stability properties [2,13].

Remark 2 In the standard nodal spectral element method, $\phi_{i}$ would be a Lagrange polynomial through the GLL points (rather than an orthonormal expansion). In practice, the same set of points is often used for both the quadrature and the nodal points of the expansion. In this case, the last term in Eq. 2 will not be zero even when a summation at $k=0$ is performed, which represents the mean or constant contribution of the nonlinear term. However, the error in this conservative term will, however, be consistent with the 
overall approximation. Standard finite-volume methods based on quadrature may also introduce this type of conservation error.

To further demonstrate how insufficient integration influences the modes, consider the following "worstcase" numerical example. Suppose that $P=10$ (i.e., 10th degree polynomials) and that the function we are trying to project is given by $u(x)=\sum_{i=0}^{10} \phi_{i}(\xi)$ - which amounts to all the modal coefficients $\hat{u}_{i}$ being set to 1.0 and mimics a case in which an element has significantly under-resolved the solution within the element. The exact Galerkin projection yielding the modes $\hat{w}_{k}, k=0, \ldots, P$ can be computed when $Q=17$ GLL points/weights are used to approximate the integral $[\cdot, \cdot]_{Q}$ given by Eq. 1 . Suppose, however, that only $Q=12 \mathrm{GLL}$ points/weights are used (sufficient for integrating a polynomial in $\mathcal{P}^{2 P}$ exactly). Instead of arriving at $\hat{w}_{k}$, we instead obtain $\tilde{w}_{k}$ as given in Eq. 2 . For this particular example, the difference between the true and the approximate inner product of the triplet is negative for each $k$. Combining this fact with the observation that $\hat{u}_{i} \hat{u}_{j}=1 \forall i, j$ in this example, we find that there is additional energy added to every mode but the mean mode (i.e., $k=0$ ). In Fig. 1 , we present a chart of the magnitude of the modal energies when reduced quadrature $\left(\tilde{w}_{k}\right)$ and exact quadrature $\left(\hat{w}_{k}\right)$ are adopted. Note that modification of the integration rule used (e.g. using Gauss-Legendre as opposed to Gauss-Lobatto-Legendre) or different choices of function may lead to different aliasing characteristics (such as energy removal.)

\section{Mathematical extensions to triangles}

In this section, we briefly review tensor-product integration on triangular domains, and then present two different means of expressing polynomials over triangles: orthogonal expansions and symmetric nodal expansions. These two types of expansions motivate different approaches to evaluating a collocation projection of a nonlinear term. We will then present an analysis of how aliasing errors can arise when insufficient integration is applied to the collocated nonlinear term.

\subsection{Integration on triangles}

As all straight-sided triangles can be affinely mapped to one another, for the purposes of this paper, we will only consider expansions on the right-sided reference triangle:

$\mathcal{T}=\left\{\left(\xi_{1}, \xi_{2}\right) \mid-1 \leq \xi_{1}, \xi_{2}, \xi_{1}+\xi_{2} \leq 0\right\}$

Fig. 1 Magnitude of the modal coefficients when exact quadrature $(Q=17)$ and reduced quadrature $(Q=12)$ is used. In this example, energy is added due to insufficient quadrature to all but the mean mode (i.e., $k=0$ )

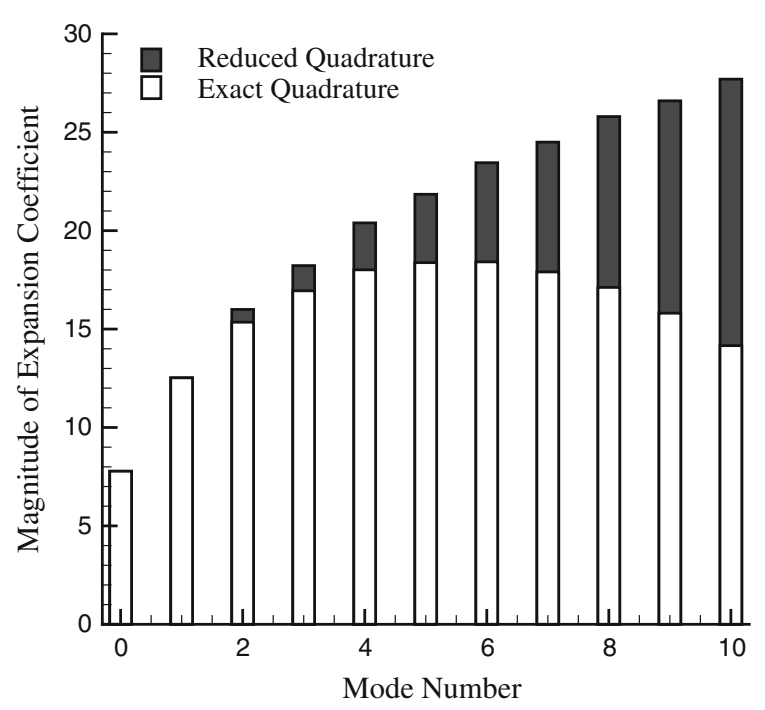



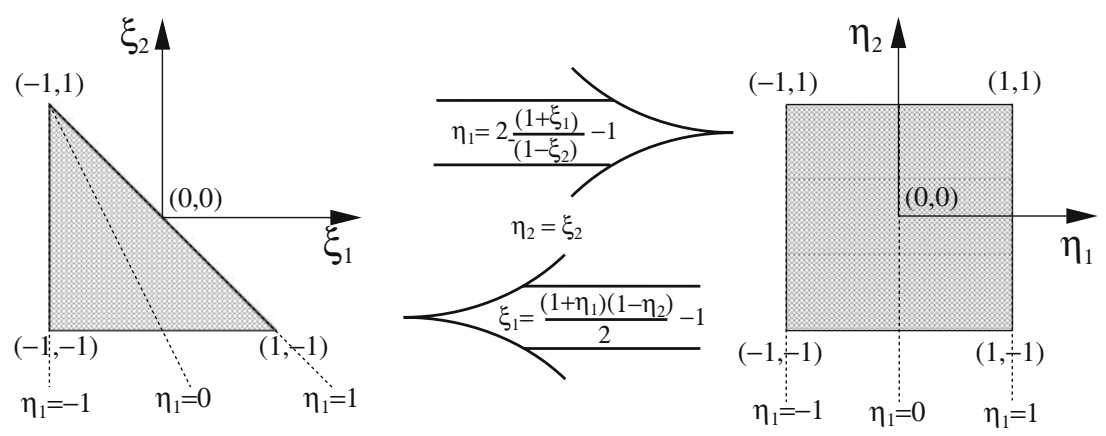

Fig. 2 Triangle to rectangle transformation

The following mapping, graphically depicted in Fig. 2, allows us to map the triangular domain to the unit square for the purposes of integration [6];

$$
\begin{aligned}
& \left(\begin{array}{l}
\eta_{1} \\
\eta_{2}
\end{array}\right)=\chi(\vec{\xi})=\left(\begin{array}{c}
2\left(\frac{1+\xi_{1}}{1-\xi_{2}}\right)-1 \\
\xi_{2}
\end{array}\right), \\
& \left(\begin{array}{l}
\xi_{1} \\
\xi_{2}
\end{array}\right)=\chi^{-1}(\vec{\eta})=\left(\frac{\left(1+\eta_{1}\right)\left(1-\eta_{2}\right)}{2}-1\right) .
\end{aligned}
$$

This bijection mapping is well behaved as we approach the singular vertex [6]. As such, it provides us with a convenient means of mapping the triangle to a domain where tensor product integration can be performed. The coordinates $\left(\eta_{1}, \eta_{2}\right)$ within the triangular domains will be referred to as the collapsed-coordinate system.

Using the above transformation, we can define the inner product over the triangle as

$$
\begin{aligned}
(f, g) & =\int_{\mathcal{T}} f(\vec{\xi}) g(\vec{\xi}) \mathrm{d} \vec{\xi} \\
& =\int_{-1}^{1} \int_{-1}^{1} \tilde{f}\left(\eta_{1}, \eta_{2}\right) \tilde{g}\left(\eta_{1}, \eta_{2}\right)\left(\frac{1-\eta_{2}}{2}\right) \mathrm{d} \eta_{1} \mathrm{~d} \eta_{2}
\end{aligned}
$$

where $\tilde{f}\left(\eta_{1}, \eta_{2}\right)=\tilde{f}(\vec{\eta})=f\left(\chi^{-1}(\vec{\eta})\right)$ (and similarly for $\tilde{g}$ ), and the term $\left(\frac{1-\eta_{2}}{2}\right)$ denotes the Jacobian of the mapping. Once transformed to a reference square, we can once again employ the Gaussian quadrature to define the following numerical approximation of the inner product

$[f, g]_{\left(Q_{1}, Q_{2}\right)}=\sum_{i=1}^{Q_{1}} w_{i}\left\{\sum_{j=1}^{Q_{2}} w_{j} \tilde{f}\left(\eta_{1 i}, \eta_{2 j}\right) \tilde{g}\left(\eta_{1 i}, \eta_{2 j}\right)\left(\frac{1-\eta_{2 j}}{2}\right)\right\}$,

where $\eta_{1 i}, \eta_{2 j}$ are the quadrature points in the $\eta_{1}$ - and $\eta_{2}$-directions. In this construction, the weights $w_{i}$ and $w_{j}$ used in Eq. 7 now correspond to any standard Gauss-Legendre rule which does not necessarily include the end-points.

Remark 3 The accuracy of the quadrature approximation is tacitly dependent on the mapping, as the quadrature rules are being applied to $\tilde{f}$ and $\tilde{g}$, and hence the residual will be expressed in terms of $\left(\eta_{1}, \eta_{2}\right)$ derivatives of the integrand. For arbitrary functions $f\left(\xi_{1}, \xi_{2}\right)$ and $g\left(\xi_{1}, \xi_{2}\right)$ on the triangle, the error terms will involve $\left(\xi_{1}, \xi_{2}\right)$-derivatives multiplied by appropriate terms from the Jacobian of the mapping. 
Note that if both $f(\vec{\xi})$ and $g(\vec{\xi})$ are separable in the collapsed coordinates (i.e., if $\tilde{f}\left(\eta_{1}, \eta_{2}\right)=\tilde{f}_{a}\left(\eta_{1}\right) \cdot \tilde{f}_{b}\left(\eta_{2}\right)$ and similarly for $\tilde{g}$ ), then we can further simplify the expression above to

$$
\begin{aligned}
{[f, g]_{\left(Q_{1}, Q_{2}\right)} } & =\sum_{i=1}^{Q_{1}} w_{i}\left\{\sum_{j=1}^{Q_{2}} w_{j} \tilde{f}_{a}\left(\eta_{1 i}\right) \tilde{f}_{b}\left(\eta_{2 j}\right) \tilde{g}_{a}\left(\eta_{1 i}\right) \tilde{g}_{b}\left(\eta_{2 j}\right)\left(\frac{1-\eta_{2 j}}{2}\right)\right\} \\
& =\left[\tilde{f}_{a}, \tilde{g}_{a}\right]_{1, Q_{1}} \cdot\left[\tilde{f}_{b}, \tilde{g}_{b}\right]_{2, Q_{2}},
\end{aligned}
$$

where $[\cdot, \cdot]_{1, Q_{1}}$ and $[\cdot, \cdot]_{2, Q_{2}}$ denote quadrature in the $\eta_{1}$ - and $\eta_{2}$-directions, respectively, given by

$\left[\tilde{f}_{a}, \tilde{g}_{a}\right]_{1, Q_{1}}=\sum_{i=1}^{Q_{1}} w_{1 i} \tilde{f}_{a}\left(\eta_{1 i}\right) \tilde{g}_{a}\left(\eta_{1 i}\right)$,

$\left[\tilde{f}_{b}, \tilde{g}_{b}\right]_{2, Q_{2}}=\sum_{j=1}^{Q_{2}} w_{2 j} \tilde{f}_{b}\left(\eta_{2 j}\right) \tilde{g}_{b}\left(\eta_{2 j}\right)\left(\frac{1-\eta_{2 j}}{2}\right)$.

Following [6], we take $[\cdot, \cdot]_{1, Q_{1}}$ to be the Gauss-Lobatto-Legendre quadrature, and $[\cdot, \cdot]_{2, Q_{2}}$ to be the Gauss-Radau-Jacobi quadrature with $\alpha=1, \beta=0$ weight distribution in the $\eta_{2}$-direction, so that the Jacobian of the mapping is handled implicitly as part of the quadrature rule up to a constant of $1 / 2$.

Remark 4 When the integrand in collapsed coordinates is separable, the quadrature error of the twodimensional integral can be determined from the error terms of the one-dimensional analysis.

In Fig. 3 (left), we present the quadrature point distribution sufficient for integrating a polynomial of degree $P=11$ on $\mathcal{T}$ exactly (to machine precision.) A Gauss-Lobatto-Legendre distribution has been applied in the " $\eta_{1}$ "-direction and a Gauss-Radau-Jacobi distribution was used in the " $\eta_{2}$ "-direction.

\subsection{Orthogonal expansions on triangles}

Since we are interested in polynomial expansions on triangles, we start by defining our polynomial space on the domain $\mathcal{T}$ as:

$\mathcal{P}^{P}=\left\{\xi_{1}^{i} \xi_{2}^{j} \mid 0 \leq i+j \leq P\right\}$.

Although the monomial form used above is convenient notationally, it is not often used in practice as the expansion rapidly becomes linearly dependent. Following [6], we define an orthogonal basis $\left\{\Phi_{i}(\vec{\xi})\right\}$
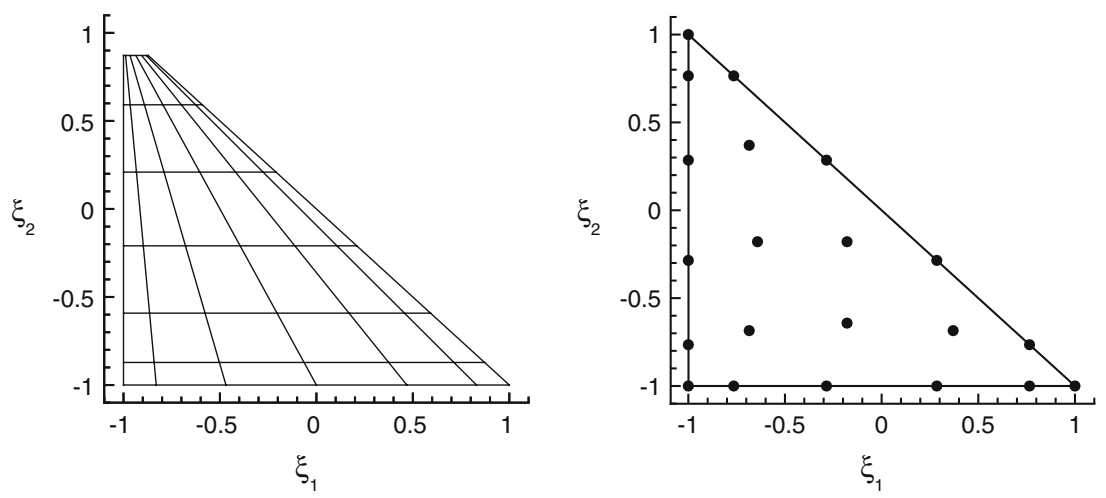

Fig. 3 Left: Collapsed coordinate quadrature points in the standard triangular $\mathcal{T}$. In the " $\eta_{1}$ "-direction a Gauss-LobattoLegendre distribution has been used and in the " $\eta_{2}$ "-direction a Gauss-Radau-Jacobi distribution was used. Right: Electrostatic nodal points distribution for $P=5$ 
consisting of $N_{P}=(P+1)(P+2) / 2$ basis functions which spans $\mathcal{P}^{P}$. The basis functions $\Phi_{i}(\vec{\xi})$, which are Sturm-Liouville approximations on a triangle, can be expressed as follows:

$\Phi_{i}(\vec{x})=\Phi_{\sigma\left(i_{1}, i_{2}\right)}(\vec{\xi})=\phi_{i_{1} i_{2}}\left(\xi_{1}, \xi_{2}\right)=\tilde{\psi}_{i_{1}}^{a}\left(\eta_{1}\right) \cdot \tilde{\psi}_{i_{1}, i_{2}}^{b}\left(\eta_{2}\right)$,

where $i=\sigma\left(i_{1}, i_{2}\right)$ denotes a bijective index mapping, and $\tilde{\psi}_{i_{1}}^{a}$ and $\tilde{\psi}_{i_{1}, i_{2}}^{b}$ denote principal functions in the collapsed coordinates (i.e., in $\eta_{1}$ and $\eta_{2}$, respectively) under the mapping $\vec{\xi}=\chi^{-1}(\vec{\eta})$.

The principal functions can be expressed in terms of Jacobi polynomials $P^{\alpha, \beta}[6]$ as follows

$\tilde{\psi}_{i_{1}}^{a}\left(\eta_{1}\right) \cdot \tilde{\psi}_{i_{1}, i_{2}}^{b}\left(\eta_{2}\right)=P_{i_{1}}^{0,0}\left(\eta_{1}\right) \cdot\left(\frac{1-\eta_{2}}{2}\right)^{i_{1}} P_{i_{2}}^{2 i_{1}+1,0}\left(\eta_{2}\right)$

Remark 5 Two key observations from the expression above emerge:

(1) The basis functions, $\phi_{i_{1}, i_{2}}$, on $\mathcal{T}$ are separable in the collapsed coordinates.

(2) The principal functions, $\tilde{\psi}_{i_{1}}^{a}, \tilde{\psi}_{i_{1}, i_{2}}^{b}$ in the collapsed coordinates remain polynomials [6].

The first observation allows for fast tensor-product integration, as discussed in Sect. 3.1. The second observation allows us to choose quadrature such that the integrals are exact to machine precision (or, in the case of insufficient quadrature, to understand the error terms as in the one-dimensional case.)

Hence, we can express any function $u \in \mathcal{P}^{P}$ in the following form:

$$
\begin{aligned}
u\left(\xi_{1}, \xi_{2}\right) & =\sum_{i=0}^{N_{P}-1} \hat{u}_{i} \Phi_{i}(\vec{\xi}) \\
& =\sum_{i_{1}=0}^{P} \sum_{i_{2}=0}^{i_{1}} \hat{u}_{\sigma\left(i_{1}, i_{2}\right)} \phi_{i_{1}, i_{2}}\left(\xi_{1}, \xi_{2}\right) \\
& =\sum_{i_{1}=0}^{P} \sum_{i_{2}=0}^{i_{1}} \hat{u}_{\sigma\left(i_{1}, i_{2}\right)} \tilde{\psi}_{i_{1}}^{a}\left(\eta_{1}\right) \tilde{\psi}_{i_{1}, i_{2}}^{b}\left(\eta_{2}\right) .
\end{aligned}
$$

Just as in the one-dimensional case, we consider a function $w \in \mathcal{P}^{P}$ that represents the Galerkin projection of the function $u^{2} \in \mathcal{P}^{2 P}$ back onto the original space where $u$ resides. The Galerkin projection is given by:

$$
\begin{aligned}
& \hat{w}_{\sigma\left(k_{1}, k_{2}\right)}=\left(\gamma_{\sigma\left(k_{1}, k_{2}\right)}\right) \sum_{i_{1}=0}^{P} \sum_{j_{1}=0}^{P} \sum_{i_{2}=0}^{i_{1}} \sum_{j_{2}=0}^{j_{1}} \hat{u}_{\sigma\left(i_{1}, i_{2}\right)} \hat{u}_{\sigma\left(j_{1}, j_{2}\right)} \cdot\left[T_{1} T_{2}\right] \\
& T_{1}=\left(\int_{-1}^{1} \tilde{\psi}_{i_{1}}^{a}\left(\eta_{1}\right) \tilde{\psi}_{j_{1}}^{a}\left(\eta_{1}\right) \tilde{\psi}_{k_{1}}^{a}\left(\eta_{1}\right) \mathrm{d} \eta_{1}\right) \\
& T_{2}=\left(\int_{-1}^{1} \tilde{\psi}_{i_{1}, i_{2}}^{b}\left(\eta_{2}\right) \tilde{\psi}_{j_{1}, j_{2}}^{b}\left(\eta_{2}\right) \tilde{\psi}_{k_{1}, k_{2}}^{b}\left(\eta_{2}\right) \frac{1-\eta_{2}}{2} \mathrm{~d} \eta_{2}\right)
\end{aligned}
$$

where

$\gamma_{i}=1 /\left(\int_{\mathcal{T}}\left[\Phi_{i}\left(\xi_{1}, \xi_{2}\right)\right]^{2} \mathrm{~d} \vec{\xi}\right)$.

Just as in Sect. 2, we can also consider what happens if insufficient quadrature is used. Taking advantage of the separability, we can determine the relation between the aliased modes $\tilde{w}_{k}$ and the true projection $\hat{w}_{k}$ as

$\tilde{w}_{\sigma\left(k_{1}, k_{2}\right)}=\hat{w}_{\sigma\left(k_{1}, k_{2}\right)}-\left(\gamma_{\sigma\left(k_{1}, k_{2}\right)}\right) \sum_{i_{1}=0}^{P} \sum_{j_{1}=0}^{P} \sum_{i_{2}=0}^{i_{1}} \sum_{j_{2}=0}^{j_{1}} \hat{u}_{\sigma\left(i_{1}, i_{2}\right)} \hat{u}_{\sigma\left(j_{1}, j_{2}\right)} \cdot\left(\tilde{T}_{2} E_{1}+\tilde{T}_{1} E_{2}+E_{1} E_{2}\right)$ 
where $\tilde{T}_{1}=\left[\tilde{\psi}_{i_{1}}^{a}\left(\eta_{1}\right) \tilde{\psi}_{j_{1}}^{a}\left(\eta_{1}\right), \tilde{\psi}_{k_{1}}^{a}\left(\eta_{1}\right)\right]_{a, Q_{1}}$, with corresponding quadrature error $E_{1}\left(i_{1}, j_{1}, k_{1}\right)$ and $\tilde{T}_{2}=$ $\left[\tilde{\psi}_{i_{1}, i_{2}}^{b}\left(\eta_{2}\right) \tilde{\psi}_{j_{1} \cdot j_{2}}^{b}\left(\eta_{2}\right), \tilde{\psi}_{k_{1}, k_{2}}^{b}\left(\eta_{2}\right)\right]_{b, Q_{2}}$, with corresponding quadrature error $E_{2}\left(i_{1}, i_{2}, j_{1}, j_{2}, k_{1}, k_{2}\right)$. Following [2], we can fix $Q_{1}$ and $Q_{2}$ so that the error terms are zero. However, in practice, $Q_{1}$ and $Q_{2}$ are chosen so that an integrand in $\mathcal{P}^{2 P}$ can be integrated exactly, but not an integrand in $\mathcal{P}^{3 P}$.

Remark 6 As long as the quadrature order is chosen so that an integrand in $\mathcal{P}^{2 P}$ is computed exactly, the mean, (or conservation), mode is not influenced by aliasing (i.e., $\tilde{w}_{0}=\hat{w}_{0}$ ). This is because, when $k_{1}=k_{2}=0$, the integrals are evaluated exactly. See also Sect. 4.1.

\subsection{Nodal expansions on triangles}

An alternative to the orthogonal expansion discussed in Sect. 3.2 is a nodal basis which spans the same polynomial space (such as the Fekete points [7], electrostatic points [8] or triangular Lobatto points [9]). These point sets are determined through a process that attempts to minimize the Lebesgue constant [7, 8, 14], a number that is an interpolation quality indicator based upon the $L_{\infty}$ norm.

A nodal basis that spans $\mathcal{P}^{P}$ can be defined as follows: Given a non-overlapping set of $N_{p}$ points $\vec{\xi}_{j} \in \mathcal{T}$ which we assume are solvable in $\mathcal{P}^{P}$, let $h_{i}\left(\xi_{1 j}, \xi_{2 j}\right)$ denote the Lagrange interpolating polynomial centred at $\vec{\xi}_{i}$ such that $h_{i}\left(\xi_{1 j}, \xi_{2 j}\right)=\delta_{i j}$. Given a function $v(\vec{\xi})$, we can define the interpolation projection $\mathcal{I} v$ of $v$ onto $\mathcal{P}^{P}$ as

$\mathcal{I} v\left(\xi_{1}, \xi_{2}\right)=\sum_{i=0}^{N_{p}-1} v\left(\xi_{1 i}, \xi_{2 i}\right) h_{i}\left(\xi_{1}, \xi_{2}\right)$.

If $v \in \mathcal{P}^{P}$, then $v=\mathcal{I} v$, and hence any function $u \in \mathcal{P}^{P}$ can be expressed in the form

$u\left(\xi_{1}, \xi_{2}\right)=\sum_{i=0}^{N_{p}-1} u\left(\xi_{1 i}, \xi_{2 i}\right) h_{i}\left(\xi_{1}, \xi_{2}\right)$.

In Fig. 3 (right), we present the electrostatic points for $P=5$. Note the symmetric geometric distribution (as compared to the distribution exhibited by the quadrature points shown in Fig. 3 (left).) Once these nodal points have been defined, it is numerically efficient to evaluate the nonlinear products using the nodal locations as collocation points, thereby making use of the Kronecker delta property of the nodal expansion. Our purpose in investigating the nodal bases is to ascertain whether evaluation of nonlinear products at a geometrically symmetric collection of points leads to a more favourable aliasing error (in terms of magnitude or error distribution) as compared to quadrature collocation technique discussed in Sect. 3.2. Since, however, we can transform from one basis to another, both types of collocation projection are possible in either expansion. Nevertheless, computational efficiency dictates that using the nodal points in conjunction with the nodal expansion would be advantageous.

Consider once again the following problem: Given a function $u \in \mathcal{P}^{P}$, find $w \in \mathcal{P}^{P}$ which represents the Galerkin projection of the function $u^{2} \in \mathcal{P}^{2 P}$ back on to the original space where $u$ resides. Using the previously discussed orthogonal basis, we can express $u^{2}$ as

$\left[u\left(\xi_{1}, \xi_{2}\right)\right]^{2}=\mathcal{I}\left(u^{2}\right)+\sum_{j=0}^{N_{2 P}-1} \hat{v}_{j} \Phi_{j}\left(\xi_{1}, \xi_{2}\right)$,

where the difference between the interpolated function and the true $u^{2}$ is expressed in terms of an orthogonal expansion with modal energy $\hat{v}_{j}$. Given Eq. 13, we can determine the relation between the modes generated using the interpolation projection $\tilde{w}_{k}$ and the true Galerkin projection $\hat{w}_{k}$. Our starting point is to recall the definition of $\hat{w}_{k}$ as the Galerkin projection of $u^{2}$, and use Eq. 13 as:

$\tilde{w}_{k}=\gamma_{k}\left(\mathcal{I}\left(u^{2}\right), \Phi_{k}\right)$, 
where we assume sufficient quadrature order to represent a function in $\mathcal{P}^{2 P}$ (otherwise the exact integrals would be replaced by quadrature rules.)

Now using Eq. 13 in the definition of $\hat{w}_{k}$, we have

$$
\begin{aligned}
\hat{w}_{k} & =\gamma_{k}\left(u^{2}, \Phi_{k}\right) \\
& =\gamma_{k}\left(\left(\mathcal{I}\left(u^{2}\right), \Phi_{k}\right)+\left(\sum_{j=0}^{N_{2 P}-1} \hat{v}_{j} \Phi_{j}, \Phi_{k}\right)\right) \\
& =\tilde{w}_{k}+\gamma_{k} \sum_{j=0}^{N_{2 P}-1} \hat{v}_{j}\left(\Phi_{j}\left(\xi_{1}, \xi_{2}\right), \Phi_{k}\left(\xi_{1}, \xi_{2}\right)\right) \\
& =\tilde{w}_{k}+\hat{v}_{k},
\end{aligned}
$$

or equivalently

$\tilde{w}_{k}=\hat{w}_{k}-\hat{v}_{k}$

where $\gamma_{k}$ was defined in Eq. 11.

Remark 7 The sum is over all $N_{2 P}$ coefficients. If the interpolation projection were replaced with an $L_{2}$ projection similar to that discussed in Sect. 3.2, the sum would only involve terms orthogonal to $\mathcal{P}^{P}$. As the interpolation projection is designed to provide optimal interpolation properties (in the $L_{\infty}$ sense), it is probable that $\hat{v}_{j}, j=0, \ldots, N_{2 P}$ will be non-zero.

One might ask whether there is any guarantee that $\hat{v}_{0}=0$, which is required for the interpolation projection to be conservative. To investigate this question, we note that the Lagrange basis can be re-written in terms of the orthogonal basis [14]:

$h_{i}\left(\xi_{1}, \xi_{2}\right)=\sum_{j=0}^{N_{p}-1} \alpha_{i j} \Phi_{j}\left(\xi_{1}, \xi_{2}\right)$,

where $\alpha_{i j}$ denotes a basis transformation matrix, which is the inverse of the generalized Vandermonde matrix as presented by [14].

Taking advantage of expression (14), we can express $u \in \mathcal{P}^{P}$ as:

$u\left(\xi_{1}, \xi_{2}\right)=\sum_{i=0}^{N_{p}-1} u\left(\xi_{1 i}, \xi_{2 i}\right)\left[\sum_{j=0}^{N_{p}-1} \alpha_{i j} \Phi_{j}\left(\xi_{1}, \xi_{2}\right)\right]$.

In this form, we can investigate what is required for the interpolation projection of $u^{2}$ to be conservative. For conservation, we want the following expression to hold:

$\int_{\mathcal{T}} u^{2} \mathrm{~d} \vec{\xi}=\int_{\mathcal{T}} \mathcal{I}\left(u^{2}\right) \mathrm{d} \vec{\xi}$

Using $\Phi_{0}(\vec{\xi})=1$ and definition (15), we obtain

$$
\begin{aligned}
\int_{\mathcal{T}} \mathcal{I}\left(u^{2}\right) \mathrm{d} \vec{\xi} & =\int_{\mathcal{T}} \sum_{i=0}^{N_{p}-1} u\left(\xi_{1 i}, \xi_{2 i}\right)^{2}\left[\sum_{j=0}^{N_{p}-1} \alpha_{i j} \Phi_{j}\left(\xi_{1}, \xi_{2}\right)\right] \mathrm{d} \vec{\xi} \\
& =\sum_{i=0}^{N_{p}-1} u\left(\xi_{1 i}, \xi_{2 i}\right)^{2} \sum_{j=0}^{N_{p}-1} \alpha_{i j} \int_{\mathcal{T}} \Phi_{j}(\vec{\xi}) \cdot \Phi_{0}(\vec{\xi}) \mathrm{d} \vec{\xi} \\
& =\sum_{i=0}^{N_{p}-1} u\left(\xi_{1 i}, \xi_{2 i}\right)^{2} \alpha_{i 0} / \gamma_{0},
\end{aligned}
$$

where $\gamma_{0}$ was defined in Eq. 11. 
For Eq. 16 to satisfy the conservation property, it must be exactly equal to $\int_{\mathcal{T}} u^{2} \mathrm{~d} \vec{\xi}$. Hence the interpolation projection is only conservative if the point set and corresponding matrix $\alpha$ are such that they can exactly integrate functions in $\mathcal{P}^{2 P}$. The rotationally symmetric points discussed previously do not correspond to quadrature points; hence they do not provide conservative projections.

One outstanding question is whether there even exists a set of $N_{P}$ points and weights such that polynomials in $\mathcal{P}^{2 P}$ (i.e., the inner product of two polynomials, each taken from $\mathcal{P}^{P}$ ) can be integrated exactly. Using the mathematical philosophy of Gaussian integration, this would seem unlikely. The strength of Gaussian quadrature lies in matching the number of modal coefficients of the polynomial expansion to the available integration degrees of freedom (points and weights). For example, in one-dimensional quadrature there are $Q$ points and weights that provide $2 Q$ degrees of freedom to determine $2 P$ modal coefficients in the integrand. An extension of this result is a tensor product of two one-dimensional expansions (typically applied within a quadrilateral region) where there are $2 Q^{2}$ quadrature degrees of freedom that can integrate functions of the form $x^{2(Q-1)} y^{2(Q-1)}$. These polynomials are in a space of dimension $4(Q-1)^{2}$, which is even higher than the degrees of freedom of the quadrature. However, this increase in the apparent dimension that the quadrature can integrate is due to the tensor-product nature of this construction. For triangle expansion in a linear rather than bi-linear polynomial space, there would appear to be a mismatch since $N_{P}$ points and weights only provide $2 N_{P}=(P+1)(P+2)=P^{2}+3 P+2$ quadrature choices; however, polynomials in $\mathcal{P}^{2 P}$ contain $N_{2 P}=[(2 P+1)(2 P+2)] / 2=2 P^{2}+3 P+1$ modal coefficients. Thus one could conjecture that there are not enough points/weights for the integration to be carried out exactly if only a nodal point set totaling $N_{P}$ points are used.

\section{Numerical results}

In this section, we consider two examples that support the analysis of Sect. 3. In Sect. 4.1, we consider the inexact Galerkin projection of a fully energised function on to the orthogonal expansion using an inexact quadrature order, similar to the one-dimensional example in Sect. 1. In this test, we therefore consider a "worse-case" scenario that might occur. In Sect. 4.2, we refine the test to consider the inexact Galerkin projection of a single polynomial mode to highlight how the polynomial order of the mode is aliased to lower-order modes.

\subsection{Inexact Galerkin projection of quadratic nonlinearities}

We consider the polynomial function of order $P$ defined as

$$
u^{\mathrm{sol}}\left(\xi_{1}, \xi_{2}\right)=\sum_{i=0}^{P} \sum_{j=0}^{P-i}\left(\xi_{1}\right)^{i}\left(\xi_{2}\right)^{j},
$$

and project the square of $u^{\text {sol }}\left(\xi_{1}, \xi_{2}\right)$ onto the orthogonal polynomial space of order $P$. In performing this projection, we have evaluated the function $\left[u^{\mathrm{sol}}\left(\xi_{1}, \xi_{2}\right)\right]^{2}$ using either a collocation projection at quadrature points along the collapsed-coordinate system, as discussed in Sect. 3.1, or a collocation projection onto the rotationally symmetric coordinate system defined by the electrostatic points, as discussed in Sect. 3.3. In the collapsed-coordinate system, we assume that we have a $P+2$ Gauss-Lobatto-Legendre points in the $\xi_{1}$ direction and $P+1$ Gauss-Radau-Jacobi points with a $\alpha=1, \beta=0$ weights distribution in the $\xi_{2}$ direction. For the electrostatic points, we use a set of points consistent with the polynomial order $P$. Once the nonlinear product has been performed at the nodal points, we then Galerkin project the collocated function onto the orthonormal expansion using exact integration.

In Fig. 4, we show the absolute difference of the expansion coefficient obtained by exact Galerkin projection onto the orthonormal basis, and the inexact Galerkin projection using the two different collocation 

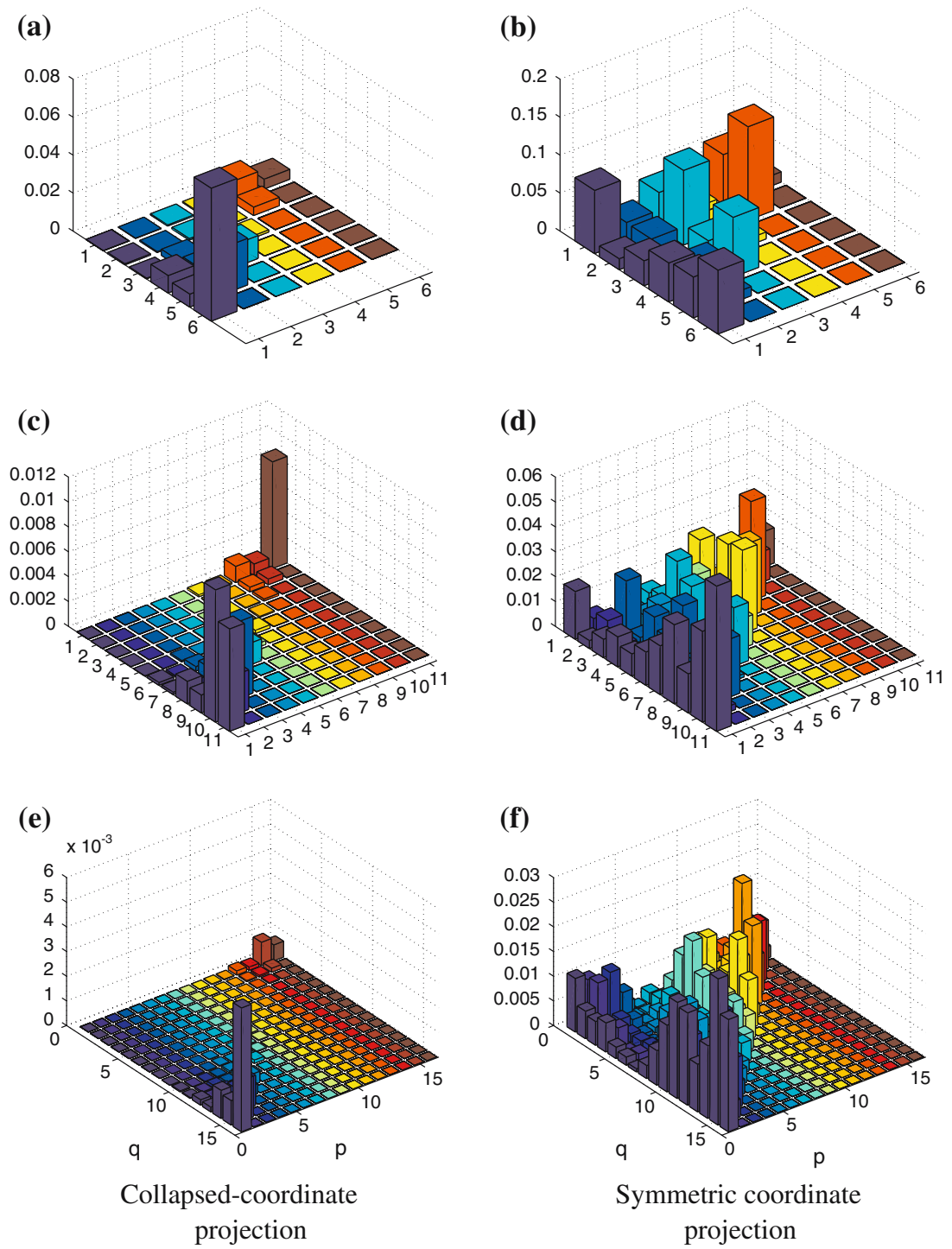

Fig. 4 Absolute difference in modal energy between exact and aliased inexact Galerkin projection $(|\hat{w}-\tilde{w}|)$ using a collocation projection at the quadrature points along the collapsed coordinates for (a) $P=5$, (c) $P=10$ and (e) $P=15$ order polynomials. Also shown is the same error using collocation at the electrostatic nodal points for $(\mathbf{b}) P=5,(\mathbf{d}) P=10$ and (f) $P=15$ order polynomials. (Note only the lower triangle of mode $p+q \leq P$ are energised in a triangular expansion.)

projections discussed previously, i.e., $\left|\hat{w}_{k}-\tilde{w}_{k}\right|$. Figures $4(\mathrm{a})$, (c) and (e) show the aliased error for the quadrature based collocation on the collapsed coordinates. Figures 4(b), (d) and (f) show the aliased error for collocation performed at the electrostatic points. We note that, for the quadrature collocation, the lower modes are not influenced significantly by the aliasing, and as previously discussed the mean mode has zero error. In contrast, the collocation at the electrostatic points introduces an aliasing error on all modes including the mean mode. The magnitude of the aliasing error in this case is also significantly larger (note the change in the $z$-axis scale.) 
4.2 Inexact Galerkin projection of a high-order orthogonal mode

In the second test, we consider the Galerkin projection of a single orthogonal mode $\phi_{p q}\left(\eta_{1}, \eta_{2}\right)$ back onto the orthogonal expansion using a fixed set of quadrature points $Q_{1}=7, Q_{2}=6$ in the $\eta_{1}, \eta_{2}$-directions. In this test, we once again have applied a Gauss-Lobatto-Legendre distribution in the $\eta_{1}$-direction and a Gauss-Radau-Jacobi with $\alpha=1, \beta=0$ distribution in the $\eta_{2}$-direction. The choice $Q_{1}=7, Q_{2}=6$ guarantees the exact evaluation of the square of a fifth order polynomial function $u\left(\xi_{1}, \xi_{2}\right)$. We note that in the $\eta_{1}$-direction the maximum polynomial order that can be exactly integrated is $2 Q_{1}-3=11$.

In Fig. 5(a-d) we show the modal energy of the (inexactly integrated) Galerkin projection in the range $0 \leq p, q ; p+q \leq 5$. As an initial validation test, Fig. 5(a) shows the energy associated with the evaluation of mode $\phi_{2,2}\left(\eta_{1}, \eta_{2}\right)$. Since this mode can be integrated exactly with the above quadrature the Galerkin projection is exact and so the evaluated mode contains only a unit energy at $p=2, q=2$.

If we now consider projection of higher polynomial modes using $\phi_{8,8}\left(\eta_{1}, \eta_{2}\right), \phi_{10,10}\left(\eta_{1}, \eta_{2}\right)$ and $\phi_{12,12}\left(\eta_{1}, \eta_{2}\right)$, there is an integration error in the Galerkin projection for some modes and so we see an aliasing error onto the lower modal energy. We note that, similar to Fig. 5(a), if exact integration were applied, we would expect to see a single mode energised with unit energy and all other modes would have a zero value. However, we observe in Fig. $5(\mathrm{~b})$ that the projection of $\phi_{8,8}\left(\eta_{1}, \eta_{2}\right)$ pollutes lower-order modes down to the mode $p=4 ; q=0$ (note the primary mode $p=8, q=8$ is not shown on the scale considered.) This aliasing pollution is to be expected as the $\eta_{1}$-quadrature can only exactly resolve integrands up to a polynomial order of $P=11$, but for this projection test one of the integrands involves the product of a $P=8$ and a $P=4$ order polynomials. Similarly, the projection of $\phi_{10,10}$ has aliased error to lower modes down to $p=2, q=0$, as shown in Fig. 5(c). When we consider the projection of an even higher polynomial mode $\phi_{12,12}\left(\eta_{1}, \eta_{2}\right)$, we observe in Fig. 5(d) that the constant mode $p=0, q=0$ becomes influenced by the aliasing.

Finally, in Figs. 5(e) and (f) we show the aliased mode $\phi_{12,12}\left(\eta_{1}, \eta_{2}\right)$ at this quadrature order and the same mode evaluated using exact integration. We observe the low-frequency form of the aliased evaluation, which highlights how the lower energy modes become polluted.

\section{Concluding remarks}

In this paper, we have analysed different approaches of evaluating quadratic nonlinear terms for spectral $/ h p$ triangular discretisations within the context of polynomial Galerkin approximations. In particular, we have considered the role of aliasing errors due to inexact quadrature associated with two different collocation projections; the first involving collocation at the quadrature points on collapsed coordinates and the second collocating at rotationally symmetric nodal points within the triangle.

The two choices of collocation points are naturally motivated by the typical expansions (collapsed coordinate modal expansion [6] and nodal, Lagrange-based expansion [7-9]) currently being adopted by spectral $/ h p$ element practitioners. However, the use of collocation projections along the rotationally nonsymmetric collapsed-coordinate system is often seen as esthetically undesirable. This observation therefore promotes the idea of introducing a collocation projection at rotationally symmetric collocation points.

During our analysis, we have focused on the error associated with the collocation projections rather than their computational cost. We note, however, that the collapsed-coordinate system allows for the sum-factorisation technique to be applied which takes advantage of a series of one-dimensional type operations [6]. Evaluation using sum-factorisation asymptotically results in an $O\left(P^{3}\right)$ cost to construct the Galerkin inner product for all expansion modes in two dimensions. The transformation to and from the rotationally symmetric nodal points, however, requires the use of a matrix operation which requires an $O\left(P^{4}\right)$ operation in two dimensions. So, for high-order polynomial expansion, using the rotationally symmetric points will necessarily be more expensive to evaluate. Nevertheless, we note that for intermediate 
(a)

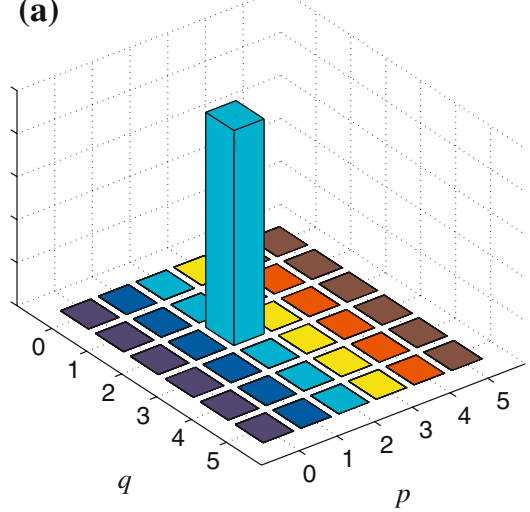

(c)

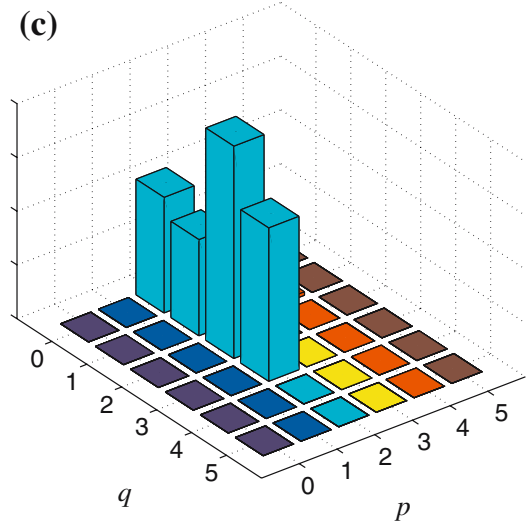

(e)

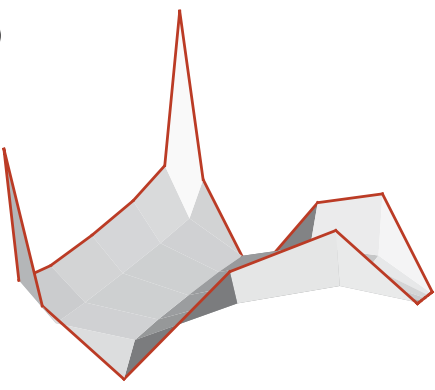

(b)

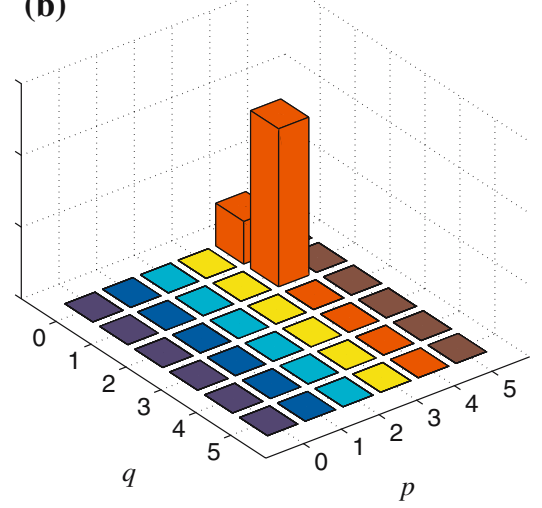

(d)

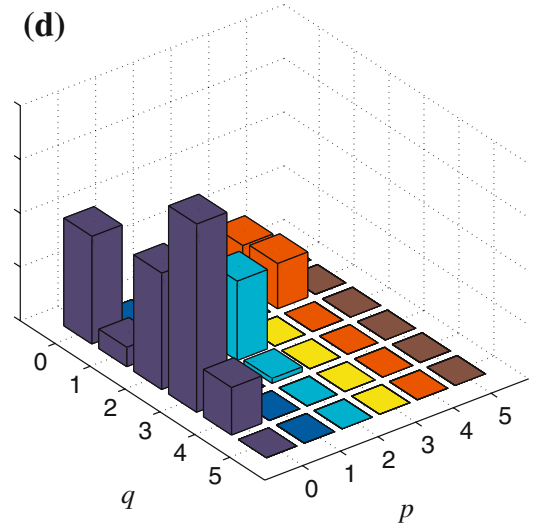

(f)

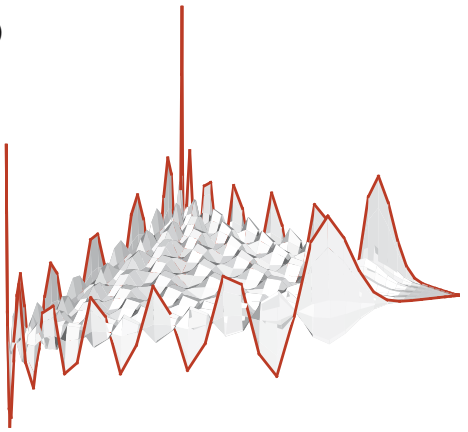

Fig. 5 Aliasing associated with the evaluation of an orthogonal expansion mode $\phi_{p, q}$ evaluated with a $Q_{1}=7, Q_{2}=6$ distribution of quadrature points: The modal energy in the fixed quadrature projection of (a) $\phi_{2,2}$, (b) $\phi_{8,8}$, (c) $\phi_{10,10}$ and (d) $\phi_{12,12}$. Also shown in (e) is the aliased approximation of $\phi_{12,12}$ where the $z$-axis scaling is arbitrary. The exact evaluation of $\phi_{12,12}$ is shown in (f)

polynomial orders, the constant terms in these evaluations can play a significant role in making the cost of the two approaches computationally similar.

In the Introduction, we raised three questions that the spectral $/ h p$ element practitioner would like to understand. We can now attempt to answers these questions as follows:

1. When is exact or consistent integration absolutely necessary?

- If one has an under-resolved simulation, then inconsistent integration will lead to the addition (or subtraction) of modal energy on higher modes of the discrete approximation. This 
addition/subtraction is clearly undesirable from both a stability and approximation point of view. Ultimately, this introduction of consistent integration is only absolutely necessary when the addition of modal energy leads to numerical instability. However, when an instability will occur, it is dependent on the type of mathematical problem being discretised and not solely on the spatial discretisation. (De-aliasing is, of course, regularly used in global spectral methods for this reason $[1])$.

\section{How does the aliasing error influence my solution?}

- For the collocation projection, at the collapsed-coordinate quadrature points, aliasing errors lead to erroneous addition or subtraction of energy primarily on the higher discretised modes. The extent of the addition depends upon the quadrature order. Quadrature order fixed at a level to exactly integrate linear terms means that the mean mode is not influenced by inexact evaluation of quadratic-type operations. In the numerical tests considered, aliasing errors were significantly reduced using this approach as compared to the rotationally symmetric collocation method. This type of collocation projection is, however, not rotationally symmetric.

- Collocation projection at rotationally symmetric nodal points leads to a higher aliasing errors that influences all modes including the mean modes, and this has implication for conservation. The truncation error from this projection will be rotationally symmetric and therefore will not be influenced by the rotation of the triangular element.

3. Is there any computationally efficient middle-ground between insufficient quadrature and exact integration?

- We have not observed any single computationally efficient middle-ground.

- When evaluating nonlinear terms that are known to be of a polynomial form, we have observed that a collocation projection at the quadrature points is desirable, since it leads to a lower aliasing error and maintains a conservation property.

- For the projection of an energetic function, which may not be a polynomial, such as initial or boundary conditions and forcing functions, the symmetry of the rotationally symmetric collocation projection is desirable. The rotationally symmetric nodal points have also been designed to minimise the $L_{\infty}$ error, which is also desirable.

We should comment that, in making our comparison between the two types of collocation, we have presumed that the projection to rotationally symmetric points is undertaken at the same order as the polynomial expansion. However, there is no reason why the expansion could not be evaluated at a set of points that would support a polynomial of higher order and then evaluate the nonlinear product. This point may well deserve further investigation, but would certainly be more computationally expensive than using the quadrature collocation projection.

Although we have focused our attention on nonlinear-type terms, we highlight that spectral $/ h p$ element approximations of deformed geometries typically involve a nonlinear mapping which introduces a polynomial approximation to the Jacobian into the integrand. Therefore, deformed-element approximation can also introduce aliasing terms, although typically during high-order mesh generation one tries to minimise the polynomial order of the Jacobian which helps to alleviate the problem [15].

Finally, we note that the aliasing error has a similar influence to backscatter in turbulence simulations where energy is coupled from the small scales back to the larger scales. This is a concept directly incorporated in some isotropic turbulence models used in large-eddy simulation (LES). In these types of simulations, almost by definition, the resolution is typically at best marginal and so a full understanding of the aliasing error before introducing the role of the turbulence model would seem to be advantageous. However, as we have seen from Eq. 12, the form of the aliasing error depends not only on the spatial contributions, but also on the modal energy of the approximations. Therefore, not only does a LES backscatter 
simulation require a dynamical turbulence model, but also the evaluation of the aliasing error needs to be determined dynamically.

Acknowledgements The first author gratefully acknowledges the partial support of NSF Career Award NSF-CCF0347791 and the computational support and resources provided by the Scientific Computing and Imaging Institute at the University of Utah. The second author would like to acknowledge the partial support of an EPSRC Advanced Research Fellowship.

\section{References}

3. Orszag S (1971) On the elimination of aliasing in finite-difference schemes by filtering high wavenumber components. J Atmos Sci 28:1074

3. Kirby RM, Karniadakis GE (2003) De-aliasing on non-uniform grids: algorithms and applications. J Comp Phys 191:249264

3. Canuto C, Quarteroni A (1982) Approximation results for orthogonal polynomials in Sobolev spaces. Math Comp 38(157):67-86

4. Canuto, C, Hussaini M, Quarteroni A, Zang T (1987) Spectral methods in fluid mechanics. Springer-Verlag, New York

5. Karniadakis G, Sherwin S (1999) Spectral/hp element methods for CFD. Oxford University Press

6. Karniadakis G, Sherwin S (2005) Spectral/hp element methods for CFD. 2nd edn. Oxford University Press, UK

7. Taylor M, Wingate B, Vincent R (2000) An Algorithm for computing Fekete points in the triangle. SIAM J Num Anal 38:1707-1720

8. Hesthaven J (1998) From electrostatics to almost optimal nodal sets for polynomial interpolation in a simplex. SIAM J Num Anal 35(2):655-676

9. Blyth M, Pozrikidis C (2006) A Lobatto interpolation grid over the triangle. IMA J Appl Math 71:153-169

10. Abramowitz M, Stegun I (1972) Handbook of mathematical functions. Dover, New York

11. Hunter D, Nikolov G (2000) On the error term of symmetric Gauss-Lobatto quadrature formulae for analytic functions. Math Comp 69:269-282

12. Lomtev I, Kirby R, Karniadakis G (1999) A discontinuous Galerkin ALE method for compressible viscous flows in moving domains. J Comp Phys 155:128-159

13. Kirby R, Karniadakis G (2001) Under-resolution and diagnostics in spectral simulations of complex-geometry flows. In: Drikakis D, Geurts B (eds) Turbulent flow computation, Kluwer Academic Publishers, The Netherlands, pp 1-42

14. Warburton T (2005) An explicit construction for interpolation nodes on the simplex. J Eng Math (accepted)

15. Sherwin S, Peiró J (2002) Mesh generation in curvilinear domains using high-order elements. Int J Num Meth Engng 53:207-223 